\title{
Multidisciplinary approach to materials selection for bipropellant thrusters using ablative and radiative cooling
}

\author{
A ADAMI $^{1}$, M MORTAZAVI $^{1, *}$ and M NOSRATOLLAHI ${ }^{2}$ \\ ${ }^{1}$ Amirkabir University of Technology, Tehran, Iran \\ ${ }^{2}$ Space Research Institute, Tehran, Iran \\ e-mail: aha.aerospace@aut.ac.ir
}

MS received 6 December 2014; revised 12 July 2015; accepted 3 August 2015

\begin{abstract}
Reduction of costs is a main consideration in every space mission, and propulsion system is an important subsystem of those missions where orbital maneuvers are considered. Lighter propulsions with higher performance are necessary to reduce the mission costs. Bipropellant propulsions have been widely used in launch vehicles and upper-stages as well as deorbit modules because of better performances in comparison with other propulsion systems. Unfortunately heat transfer and thermal control limit bipropellant propulsion performance and maximum performance cannot be achieved. Well-known cooling methods such as regenerative and film cooling increase the cost using extra equipment and high temperature materials. In this paper, a new approach for cooling is presented based on combined ablative and radiative cooling. Governing equations are derived for two or three layers of thermal protection system (TPS) to optimize the TPS mass. The first layer is used as an ablative layer to control the temperature where the second and third layers are used as an insulator to control the heat fluxes. Proposed cooling method has been applied for two real bipropellant thrusters. According to the results, the presented algorithm can suitably predict the heat fluxes and satisfy the wall temperature constraint. Then, the algorithm has been used to minimize the wall temperatures as low as possible and replace high temperature materials (platinum alloy) with common materials (composite or steel). It is shown that selection of TPS materials affects the TPS mass and Isp simultaneously, but conversely. Best solution should be derived by trading off between structure temperature (cost), Isp (performance), and TPS thicknesses (geometry). Multidisciplinary approach to TPS and structure material selection of a bipropellant thruster is presented for a case study. It has been shown that mass and performance penalties of using TPS are acceptable, considering the advantages of using steel alloy instead of platinum alloy.
\end{abstract}

Keywords. Heat transfer; multidisciplinary; bipropellant; ablation; materials selection; cooling.

\section{Introduction}

Liquid thrusters are widely used in space application, especially for orbital maneuvers and launch vehicles. New missions demand new propulsion requirements with lower design and manufacturing costs. Additionally, new materials and propellants will improve the propulsion performances and new generations of bipropellant propulsions will appear. Nowadays, modern deigning methods such as multidisciplinary design optimization (MDO) are used to derive an optimum solution. Optimization of complex engineering systems has always been an integral part of design. Due to the size and complexity of systems, the design of a whole system is broken down into multiple disciplines. All of the disciplines should be simultaneously optimized to find a global optimum. Long processing time is a disadvantage of such algorithms. In preliminary design phase, semi-accurate

*For correspondence modeling of each discipline is usually used to analyze many configurations in shorter elapsed time. One of the most important disciplines in MDO of bipropellant propulsion is thermal control. Heat transfer limits bipropellant propulsion performance. Maximum performance cannot be achieved because of wall temperature constraint. Well-known cooling methods such as regenerative and film cooling improve the performance but these common cooling methods cannot achieve maximum performance freely. A major limitation is because of using one of the propellants as a coolant. Additionally, such cooling methods increase the cost using extra equipment and high temperature materials. In this paper, a new approach of cooling is presented based on combined ablative and radiative cooling that is especially suitable for short time activity with high thrust levels. To do this, heat transfer from combustion chamber and nozzle should be rapidly predicted. 
The purpose of a thermal control of thrusters is to maintain all thrusters' components within the allowable temperature. This is only achieved by control of heat flux from a combustion chamber. Because of high combustion temperatures (up to $3700 \mathrm{~K}$ ) and high heat flux rates (peak may go beyond $160 \mathrm{MW} / \mathrm{m}^{2}$ ) from the hot gases to the chamber wall, thruster cooling is a major design consideration. Two approaches are available to encounter [1,2]. The first one is steady state method, in which the heat transfer rate through combustion wall and temperature on the wall are constant; in other words, there is a thermal equilibrium. The second method to cool combustion chamber is the unsteady state method or transient heat transfer method. For this method, there is no thermal equilibrium and the temperature on combustion wall continues to increase. The propulsion system has to be stopped before the wall temperature reaches the critical point. For continuous thrust regime in space mission, steady state methods should be provided. Regenerative cooling, film cooling, transpiration cooling, radiative cooling, and ablative cooling are generally used to control heat fluxes of combustion chamber and providing suitable structure temperatures $[3,4]$.

Regenerative cooling is the most widely used method of cooling a high performance combustion chamber and is accomplished by flowing high velocity coolant over the backside of the chamber hot gas wall to convectively cool the hot gas liner [5, 6]. The coolant, heated up by the hot liner, is then discharged into the injector and used as a propellant. Engines that have this kind of cooling are, for example, the AETUS II, RL10A, RD861K and VINCI [7].

Ablative cooling uses a particular combustion gas-side wall material that is sacrificed by melting, vaporization, and chemical changes to dissipate heat. As a result, relatively cool gases flow over the wall surface, thus lowering the boundary-layer temperature and assisting the cooling process. Radiative cooling radiates the heat away from the surface of the outer chamber walls. An example of entirely radiation-cooled thruster is the RS-21 Mariner/Viking Orbiter spacecraft.

Transpiration cooling is accomplished by introducing a coolant (gaseous or liquid) through porous chamber walls at a rate sufficient to maintain the desired combustion chamber wall temperature. This technique was applied to cool the injector faces of the J-2, RS-44 and SSME.

Film cooling protects the chamber walls from excessive heat with a thin film of coolant. Coolant is injected through orifices near the injector or near the throat with special slots. This method has been widely used, in particular for high heat fluxes [8-10]. Sample engines where film cooling is applied are the SSME, Vulcain 2, RD-171and RD-180.

Combinations of the aforementioned methods are nowadays used [11] to increase the thermal performances. Combination of regenerative and film cooling is more interesting because of good performance without activity limitation. Although comparison between cooling methods depends on thermal and structural objectives, for a space mission, selection of cooling methods is affected by propulsion mass and cost. Regenerative cooling causes considerable pressure drop in feeding lines. Thus, this method is generally proposed for turbo pump feeding systems. Turbo pumps provide high pressure, which is suitable for high propellant flow and high thrust level. Pressurized feeding is usually selected for small bipropellant thrusters, thus, regenerative cooling which needs extra equipment (piping) is not suitable. Transpiration cooling method demands additional (coolant) mass that does not seriously contribute to producing thrust and needs thruster piping again. It leads to decrease the propulsion performance and increase in propulsion mass. Radiative cooling and film cooling are mostly used for small bipropellant thrusters [4, 12]. Reduction in cost and mass for space missions leads to renew interest in simpler production procedure and lower propulsion system cost. For example for short-duration operation (up to a few seconds), uncooled chambers may be used. Chamber wall absorbs the heat until the wall temperature approaches the failure temperature (unsteady state condition). This option is usually used for solid propellant propulsions [13]. Governing equations of regenerative cooling, transpiration cooling and film cooling are well developed in the literature [3, 4, 12]. These cooling methods have a similar nature in fact. Radiative cooling is the simplest cooling method but demands high temperature martial technology. Space propulsion companies always try to develop high temperature material for combustion such as iridium, platinum and rhenium [14-16], which indicates higher cost. A general disadvantage of radiative cooling is loss of performance. High heat flux diffusion of thrust chamber wall (because of high wall temperature) causes loss of energy (temperature drop). Lower temperature is considered as lower Isp and performance.

According to the mentioned notes, heat control should be done with lowest cost and lowest performance loss. To achieve this, combinations of ablative and radiative cooling methods are proposed in this paper for lower cost and simpler production procedure of small bipropellant propulsion system.

\section{Heat transfer modeling of combustion chamber}

Heat transfer from combustion chamber and thermal modeling of bipropellant thrusters are one of the important disciplines in propulsion design algorithm. Hard coupling between thermal limitation and thruster's performance (such as pressure, mass flow, o/f ratio,...) takes place because one of the propellants (usually fuel) is simultaneously involved in different disciplines [3, 17]. Analytical and computational tools were developed for over cooled walls $[11,18]$, but ablative cooling for liquid bipropellant is difficult to find in literature.

The largest part of the transferred heat from the hot chamber gases to the chamber walls is by convection and 


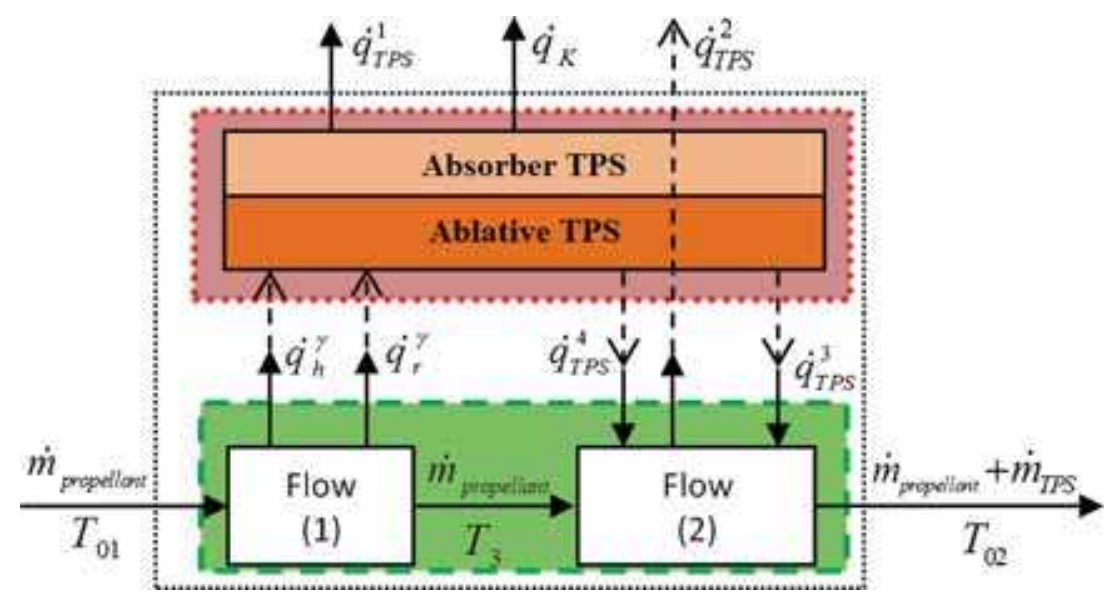

Figure 1. Thermal equilibrium for bipropellant thruster with ablative and radiative cooling.

radiation. The amount of transferred heat by conduction is small and usually negligible in preliminary design phases. Thermal equilibrium of combustion chamber can be presented as in figure 1 for combined radiative and ablative cooling.

In this model, it is considered that propellant enters the control volume with temperature $T_{01}$. Temperature of first section (Adiabatic Temperature) depends on $\mathrm{O} / \mathrm{F}$ ratio (oxidant mass by fuel mass ratio)

$$
\dot{Q}_{\text {prop }}=\dot{m}_{\text {propellant }} C_{P}^{\text {in }} T_{01}(O / F)
$$

Combustion products lose energy by convection $\dot{q}_{h}^{\gamma}$ and radiation $\dot{q}_{r}^{\gamma}$.

$$
\left\{\begin{array}{l}
\dot{q}_{h}^{\gamma}=h_{\gamma}\left(\tilde{T}-T_{S}\right) \\
\dot{q}_{r}^{\gamma}=\varepsilon_{\gamma} \sigma T_{01}^{4} \\
\sigma=5.6697 \times 10^{-8} \frac{W}{\left(m^{2} \cdot K^{4}\right)} \\
\tilde{T}=\frac{T_{01}+T_{02}}{2}
\end{array}\right.
$$

where $T_{S}$ is inner thermal protection system (TPS) wall (ablative TPS) temperature, $\tilde{T}$ is mean temperature of combustion chamber [19], $T_{02}$ is the temperature after heat transfer (real temperature), $h_{\gamma}$ is convective heat coefficient. Adami [3] proposed a modified relation (3) which needs a few inputs to derive $h_{\gamma}$ and $\varepsilon_{\gamma}$ independent of wall temperature.

$$
\begin{aligned}
\varepsilon_{\gamma} & \sim 0.1 \\
h_{\gamma} & =Z_{c} 0.5 \rho_{\text {comb }} V_{\text {flow }} C_{P} \mu^{0.3} \mathrm{Pr}=Z_{c} \frac{\dot{m}_{\text {propellant }}}{2 A} C_{P} \mu^{0.3} \mathrm{Pr} \\
C_{P} & =\frac{\gamma R}{\gamma-1}, \quad \operatorname{Pr}=\frac{4 \gamma}{9 \gamma-1}, \quad \mu=1.78 \times 10^{-7} M_{g}^{0.5} T_{01}^{0.6} \\
Z_{c} & =\frac{\pi R_{\text {comb }}^{2}}{A_{\text {lat }}}
\end{aligned}
$$

where $R$ is a constant parameter of gas combustion products, $\gamma$ is an isentropic exponent, $\operatorname{Pr}$ is Prandtl number, $\mu$ is viscosity, $M_{g}$ is molecular weight, $C_{P}$ is specific heat at constant pressure and $A$ is cross-section $A^{*}<A<A_{\text {comb }}$ of flow (port).

The chamber wall $\left(T_{S}\right)$ is warmed-up. It approaches the maximum value called ablative temperature $T_{A b l}$. Ablative TPS begins to ablate. The decomposition of ablative materials is complex, involving many and multifarious physical and chemical processes [13]. Heat absorption by ablation is presented as Eq. (4).

$$
\dot{Q}_{A b l}=\dot{m}_{A b l} C_{A b l}=\dot{q}_{T P S}^{1} A_{l a t} \Rightarrow \dot{q}_{T P S}^{1}=\rho_{A b l} \dot{r}_{A b l} C_{A b l}
$$

where $\rho_{A b l}$ is density of the ablative TPS, $\dot{r}_{A b l}$ is ablation velocity and $C_{A b l}$ is ablation heat coefficient. Some of the absorbed energy comes back to the combustion flow by radiation from hot wall and ablation mass flow at ablation temperature.

$$
\begin{aligned}
& \dot{q}_{T P S}^{3}=\dot{m}_{A b l} C_{P}^{A b l} T_{A b l} \\
& \dot{q}_{T P S}^{4}=\varepsilon_{A b l} \sigma T_{S}^{4}
\end{aligned}
$$

It should be noted that if wall temperature is lower than ablative temperature, then, ablation does not happen. Input energy of ablation mass flow $\left(\dot{q}_{T P S}^{3}\right)$ absorbs some energy again until it reaches the real combustion chamber temperature and leaves the control volume.

$$
\dot{q}_{T P S}^{2}=\dot{m}_{A b l} C_{P}^{A b l}\left(T_{02}-T_{A b l}\right)
$$

Chamber structure should be protected from high temperature. Ablative TPS keeps the inner wall temperature nearly constant, but this temperature is high enough to melt any structure. Ablative materials have generally high density and high conductive heat coefficient $\left(K_{A b s}\right)$. It is not good to use ablative material as an insulator and, hence, the second TPS layer is proposed. Conductive heat from two TPS layers is finally emitted by radiation from lateral surface of the structure.

$$
\begin{aligned}
& \dot{q}_{K}=K_{A b s} \frac{T_{A b l}-T_{s t r}}{\delta_{A b s}} \\
& \dot{q}_{r-s t r}=\varepsilon_{s t r} \sigma T_{s t r}^{4}
\end{aligned}
$$


where $\delta_{A b s}$ is the thickness of the insulator. Sometimes, $T_{A b l}$ is high enough to melt the insulator TPS. In such condition another TPS (usually ablative TPS) may be used to decrease the temperature to suitable values for the insulator TPS. For three-layer TPS, Eq. (8) is used instead of Eq. (7).

$$
\begin{aligned}
& \dot{q}_{K-2}=K_{A b s-2} \frac{T_{A b s-2}^{\max }-T_{s t r}}{\delta_{A b s-2}} \\
& \dot{q}_{K-1}=K_{A b s-1} \frac{T_{A b l}-T_{A b s-1}^{\max }}{\delta_{\delta_{A b s-1}}} \\
& \text { if Ablative TPS Selected } \\
& \dot{q}_{K}=K_{A b l} \frac{T_{A b l}-T_{A b s}^{\max -1}}{\delta_{A b s-1}} \\
& \dot{q}_{r-s t r}=\varepsilon_{s t r} \sigma T_{s t r}^{4}
\end{aligned}
$$

Under steady state condition and thermal equilibrium for propellant flow, TPS and control volume lead to Eq. (9).

$$
\left\{\begin{array}{l}
\dot{m}_{\text {propellant }} C_{P}^{i n} T_{01}+\dot{m}_{A b l} C_{P}^{A b l} T_{A b l}-A_{\text {lat }} h_{\gamma}\left(\tilde{T}-T_{A b l}\right) \\
\quad-A_{\text {lat }} \sigma\left(\varepsilon_{\gamma} \tilde{T}^{4}-\varepsilon_{A b l} T_{A b l}^{4}\right) \\
\quad=\left(\dot{m}_{A b l}+\dot{m}_{\text {propellant }}\right) C_{P}^{\text {out }} T_{02} \\
A_{\text {lat }} h_{\gamma}\left(\tilde{T}-T_{A b l}\right)+A_{\text {lat }} \sigma\left(\varepsilon_{\gamma} \tilde{T}^{4}-\varepsilon_{A b l} T_{A b l}^{4}\right) \\
\quad=\dot{m}_{A b l} C_{A b l}+A_{\text {lat }} \varepsilon_{s t r} \sigma T_{s t r}^{4} \\
\varepsilon_{s t r} \sigma T_{s t r}^{4}=\dot{q}_{K-1}=\dot{q}_{K-2}
\end{array}\right.
$$

The above three equations have four unknowns including $T_{02}, \dot{m}_{A b l}, T_{s t r}$ and $\delta_{A b s}$. This condition is suitable for optimization. Objectives can be selected as minimizing $\dot{m}_{A b l}$ or maximizing $T_{02}$, but unique answer will be derived if $T_{s t r}$ is limited. Finally, total TPS mass can be predicted by Eq. (10).

$$
\left\{\begin{array}{l}
M_{T P S}=M_{A b l}+M_{A b s-1}+M_{A b s-2} \\
M_{A b l}=\dot{m}_{A b l} \Delta t \\
M_{A b s}=\left(\delta_{A b s-1} \rho_{A b s-1}+\delta_{A b s-2} \rho_{A b s-2}\right) A_{l a t}
\end{array}\right.
$$

If ablation is not needed, Eq. (11) can be alternatively used. Unknowns are $T_{02}, \delta_{A b s}, T_{s t r}$ and $T_{s}$.

$$
\left\{\begin{array}{l}
\dot{m}_{\text {propellant }} C_{P} T_{01}-A_{\text {lat }} h_{\gamma}\left(\tilde{T}-T_{S}\right) \\
\quad-A_{\text {lat }} \sigma\left(\varepsilon_{\gamma} \tilde{T}^{4}-\varepsilon_{A b l} T_{s}^{4}\right)=\dot{m}_{\text {propellant }} C_{P} T_{02} \\
A_{\text {lat }} h_{\gamma}\left(\tilde{T}-T_{s}\right)+A_{\text {lat }} \sigma\left(\varepsilon_{\gamma} \tilde{T}^{4}-\varepsilon_{A b l} T_{s}^{4}\right)=A_{\text {lat }} \varepsilon_{s t r} \sigma T_{s t r}^{4} \\
\varepsilon_{s t r} \sigma T_{s t r}^{4}=\dot{q}_{K-1}=\dot{q}_{K-2}
\end{array}\right.
$$

According to Eqs. (9) and (11), there is a unique set of $\delta_{A b s}$ and $\dot{m}_{A b l}$ for every $T_{s t r}$. Algorithm can be used for every section through the thruster (combustion and nozzle). In this paper, two sections are considered including combustion chamber and DC nozzle. Therefore, combustion products enter with adiabatic temperature $T_{01}$ and leave the combustion chamber (and enter the nozzle) with temperature $T_{02}$.

Using the mentioned equations, materials selection, the related thicknesses of TPS can be optimized to achieve minimum TPS mass to satisfy the structure temperature constraint.

\section{Validation}

Validation of heat transfer is usually concentrated in prediction of $\dot{q}_{h}, \dot{q}_{r}$ and structure temperature in steady state condition. Radiative cooling from outer surface of the wall is only considered to solve the energy equations (compression between cooling methods will be done later). The following two cases are selected to verify the presented algorithm. Specifications of the selected thrusters are shown in table 1 .

The results are compared in figure 2 for the first case ( $T=$ $420 \mathrm{~N}$ ). It should be noted again that only radiative cooling has been considered.

The $x$-distance is measured from the head of the thruster and radiuses are scaled. According to the results, the proposed model can suitably predict the heat transfer at nozzle (Error $<15 \%$ ) and combustion chamber (Error $<13 \%$ ) in comparison with accurate numerical methods such as Bartz and Ievlev. The wall temperatures are also suitably predicted. Results of the second case $(\mathrm{T}=10 \mathrm{~N})$ are compared in figure 3.

According to the results in figure 3 , the algorithm suitably predicts the heat transfer and wall temperature at nozzle $($ Error $<16 \%)$ and at combustion chamber (Error $<14 \%)$. Thus, model can be useful for preliminary design phases and MDO algorithm.

Finally, it is understood from the above analysis that, without cooling the thruster, wall temperature is high enough to fail with every known material. Therefore, film cooling was used to decrease the wall temperature below the permitted temperature (1800 K for Platinum Alloy) in the mentioned thrusters. Results of film cooling are illustrated in figures 4 and 5 .

The $x$-distance is measured from the head of the thruster and radiuses are scaled. A numerical algorithm for film cooling has provided the data in figures 4 and 5 . The results are presented to show the fact that cooling methods can reduce the wall temperatures below than the permitted values. According to the results, shown in figures 4 and 5, wall temperatures are lower than the permitted values. In addition, it is understood that lower thrust level leads to decrease in the wall temperature. For example, the maximum value of the wall temperature is close to $1400 \mathrm{~K}$ for $\mathrm{T}=420 \mathrm{~N}$, but the maximum value is $1300 \mathrm{~K}$ for $\mathrm{T}=10 \mathrm{~N}$.

The disadvantage of the applied cooling method is the lower Isp because of the selection of $\mathrm{O} / \mathrm{F}$ ratio (equal to 1.65) lower than an optimum value $(\mathrm{O} / \mathrm{F}=2.25)$. In fact, thermal problems limit the $\mathrm{O} / \mathrm{F}$ ratio and if optimum $\mathrm{O} / \mathrm{F}$ ratio is selected then wall temperature (at throat) grows over $1600 \mathrm{~K}$

\section{Ablation and radiation cooling}

It is clear that using high temperature material (such as Platinum Alloy) increases the cost. The main goal is to decrease 
Table 1. Case studies specifications.

\begin{tabular}{|c|c|c|c|c|c|}
\hline Companies & Propellant & $\mathrm{O} / \mathrm{F}$ & Thrust & $\begin{array}{c}\text { Mass } \\
\text { flow }\end{array}$ & $\begin{array}{l}\text { Chamber } \\
\text { pressure }\end{array}$ \\
\hline & $\mathrm{MMH}-\mathrm{N}_{2} \mathrm{O}_{4}$ & 1.65 & $420 \mathrm{~N}$ & $135 \mathrm{gr} / \mathrm{s}$ & 10 bar \\
\hline & $\mathrm{MMH}-\mathrm{N}_{2} \mathrm{O}_{4}$ & 1.65 & $10 \mathrm{~N}$ & $3.5 \mathrm{gr} / \mathrm{s}$ & 9 bar \\
\hline Companies & $\begin{array}{c}\text { Throat } \\
\text { diameter }\end{array}$ & $\begin{array}{c}\text { Nozzle end } \\
\text { diameter }\end{array}$ & $\begin{array}{c}\text { Structure } \\
\text { material }\end{array}$ & $\begin{array}{l}\text { Cooling } \\
\text { method }\end{array}$ & \\
\hline
\end{tabular}

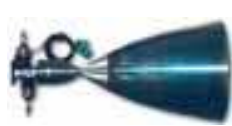

Model No S400-12

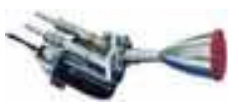

Model S 10-21 16.4mm $\quad 244 \mathrm{~mm} \quad$ Platinum alloy $\quad$ Film and Radiative

$2.85 \mathrm{~mm} \quad 35 \mathrm{~mm} \quad$ Platinum-Rhodium Film and Radiative the wall temperature while typical material (for example, Steel Alloy) can be applied.

As mentioned before, two or three TPS layers are used for simultaneous control of heat fluxes and temperatures. Properties of some thermal protection materials are summarized in table $2[20,21]$.

Although propulsion's geometry has been specified, the TPS thicknesses will change the dimensions. Thus, algorithm should be consecutively used. It is clear that dimensions of the combustion chamber or nozzle's throat will be updated. Avcoat and graphite are considered as an ablative material and silicon carbide is considered as an insulator.

Results of the combined ablative and radiative cooling for the first case study are illustrated in figure 6 and figure 7 for graphite and avcoat, respectively.

According to the results depicted in figure 6, nearconstant wall temperatures have been provided by using

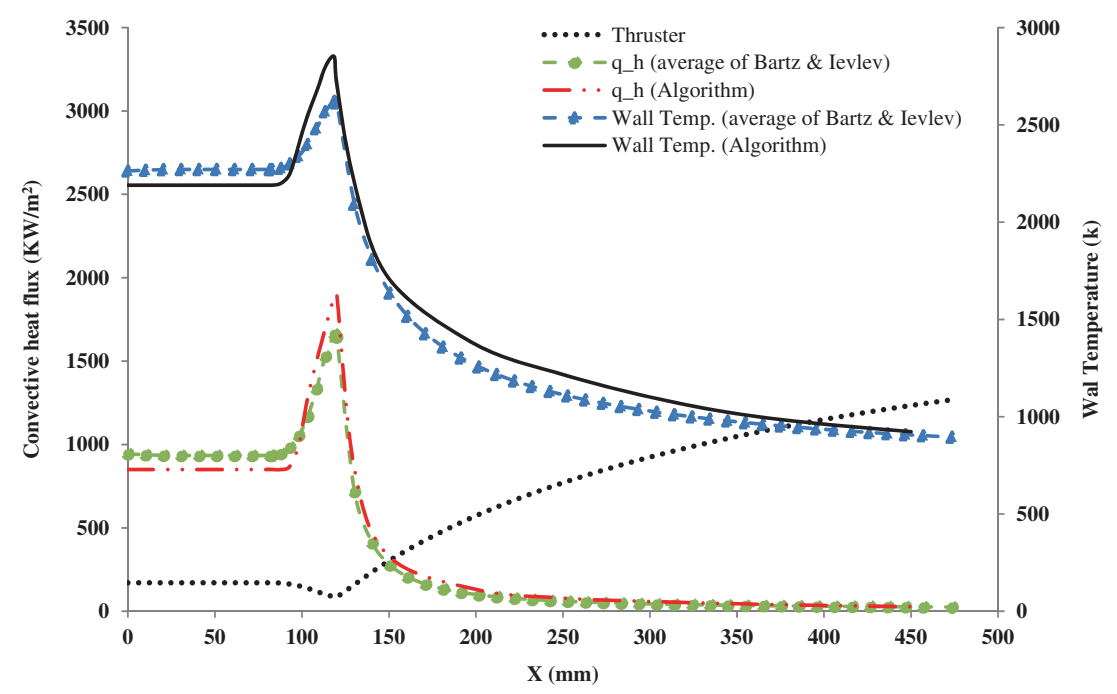

Figure 2. Wall temperature and heat flux comparison of the first case $(\mathrm{T}=420 \mathrm{~N})$. 


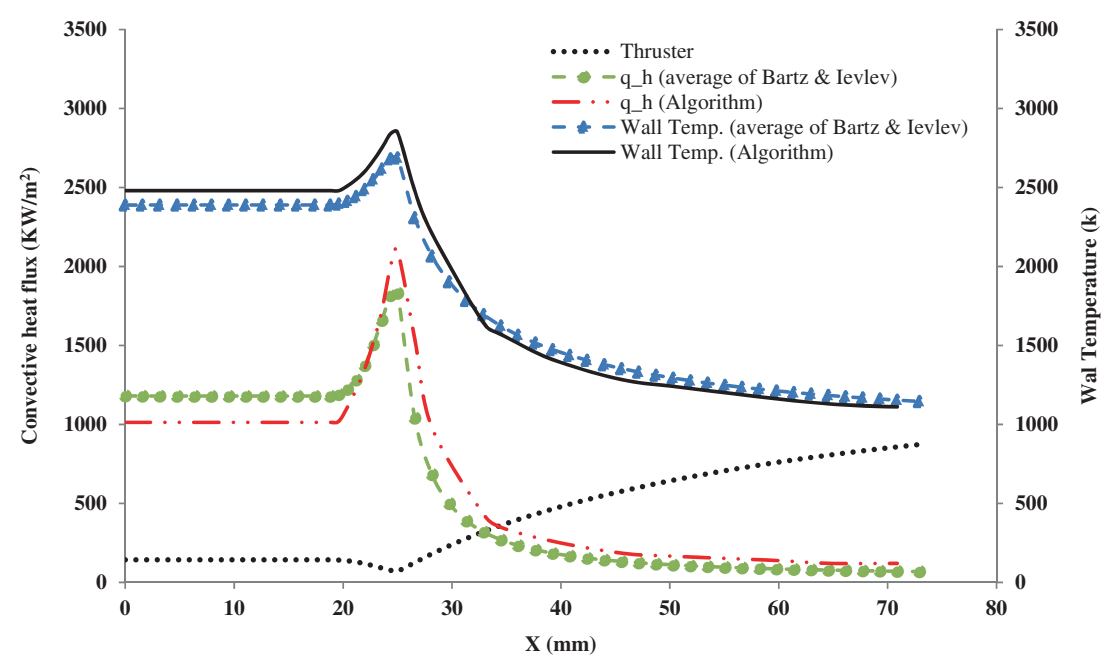

Figure 3. Wall temperature and heat flux comparison of the second case $(\mathrm{T}=10 \mathrm{~N})$.

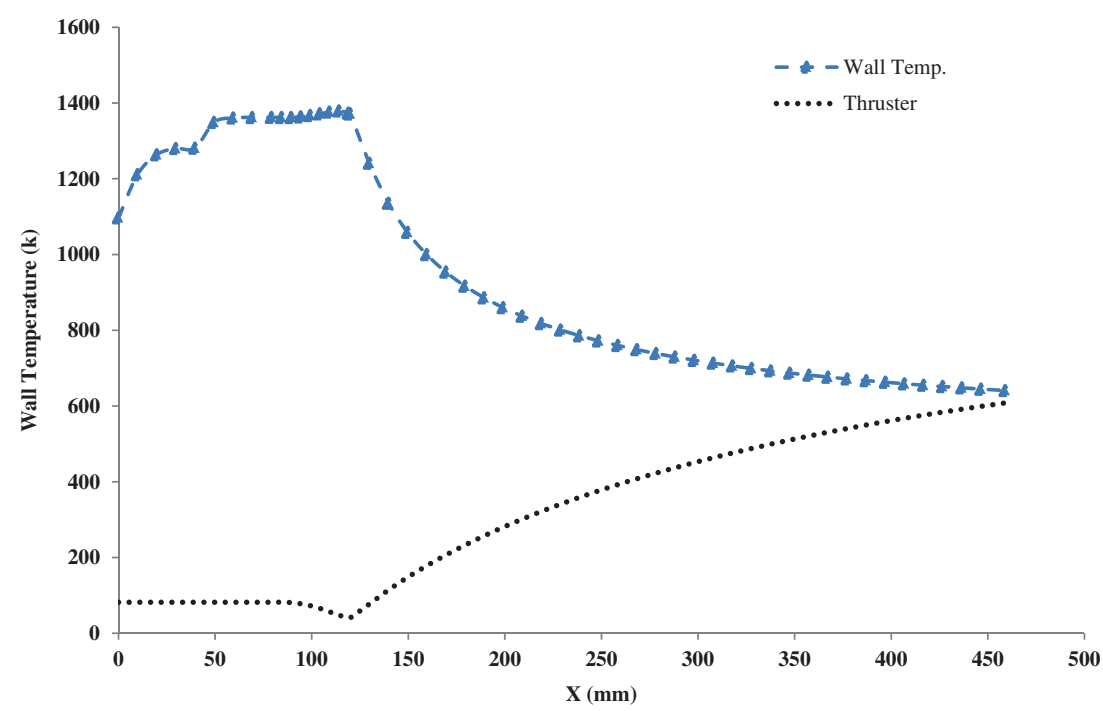

Figure 4. Wall temperature of the first case $(\mathrm{T}=420 \mathrm{~N})$ with film cooling and radiative cooling.

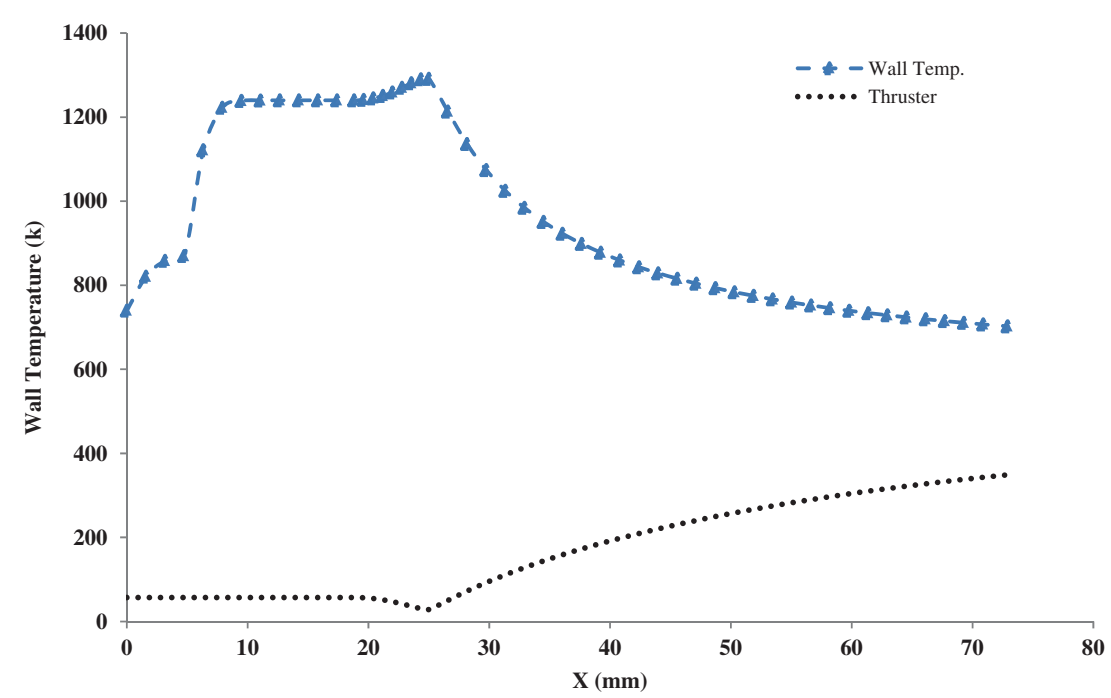

Figure 5. Wall temperature of the second case $(T=10 \mathrm{~N})$ with film cooling and radiative cooling. 
Table 2. Summary of TPS properties.

\begin{tabular}{lccccc}
\hline Material & $K_{T P S}(\mathrm{~W} / \mathrm{m} . \mathrm{K})$ & $\mathrm{Cp}(\mathrm{J} / \mathrm{kg}-\mathrm{K})$ & $\rho(\mathrm{Kg} / \mathrm{m})$ & $T_{A b l}(\mathrm{~K})$ & $C_{A b l}(\mathrm{MJ} / \mathrm{kg})$ \\
\hline Silica & 1.3 & 740 & 320 & 1685 & - \\
Silicon carbide & 0.82 & 750 & 310 & 1650 & - \\
Alumina & 0.62 & 775 & 512 & 2090 & - \\
Zirconia & 0.14 & 700 & 736 & 2480 & - \\
Graphite & 1.59 & 1631 & 1400 & 3778 & 66.8 \\
Avcoat39-5026 & 25 & 1110 & 513 & 835 & 47 \\
\hline
\end{tabular}

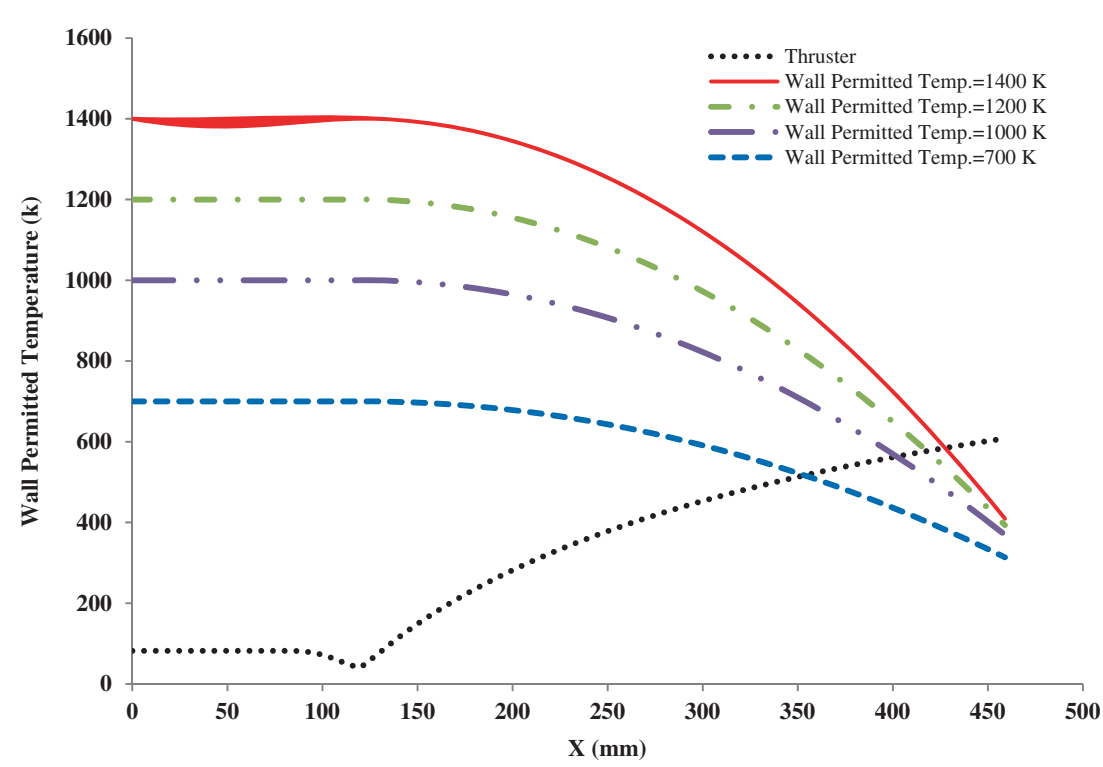

Figure 6. Design results for graphite and silicon carbide.

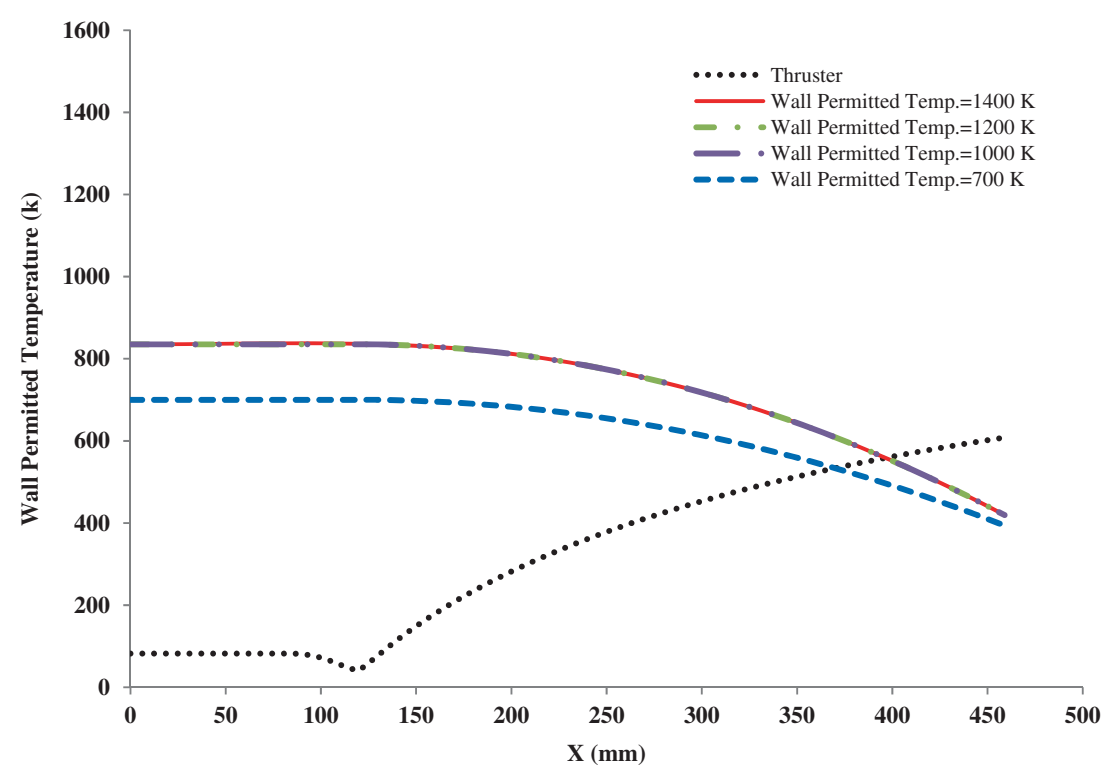

Figure 7. Design results for avcoat and silicon carbide.

graphite as an ablative TPS and silicon carbide as an insulator. As shown, every desired temperature can be provided because heat flux and wall temperature are simultaneously controlled, as mentioned before.
According to figure 7 , there is a constant wall temperature near ablation temperature for every permitted wall temperature higher than ablation temperature of avcoat. That means it is impossible to have wall temperature more 


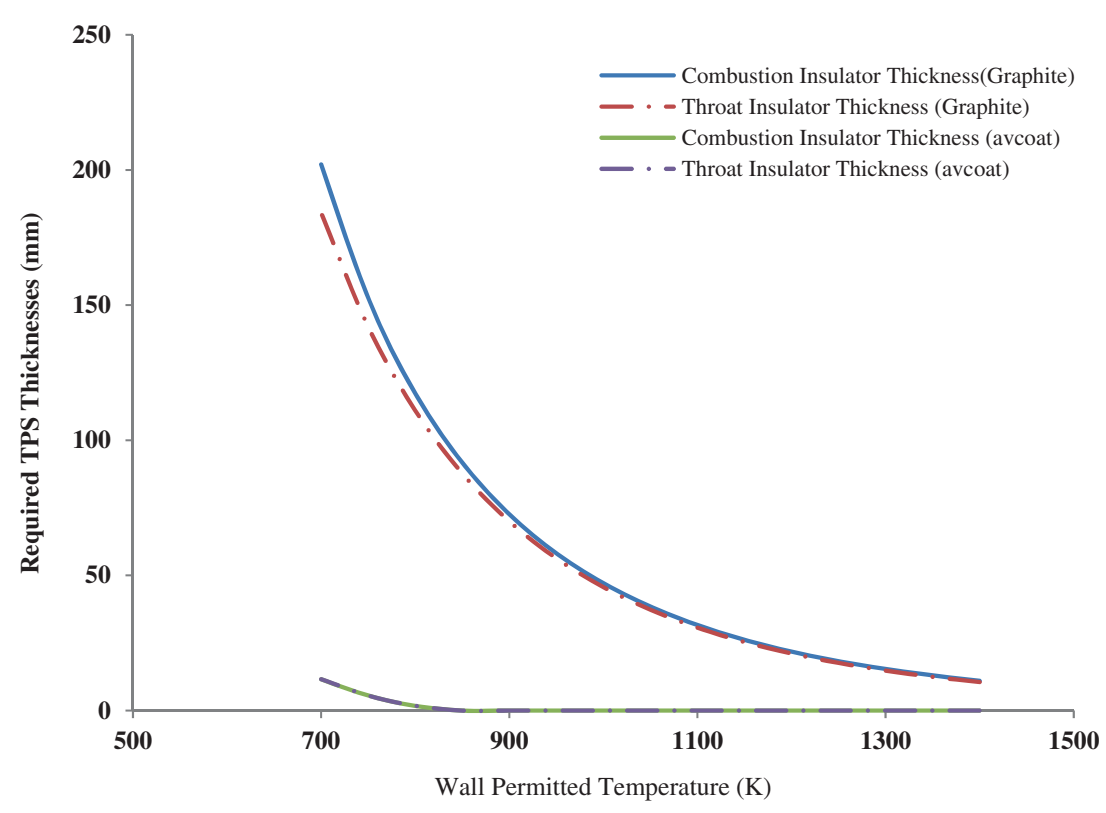

Figure 8. Comparison of required TPS thicknesses for graphite and avcoat.

than ablation temperature and ablative cooling is required for every permitted wall temperature higher than ablation temperature. Similarly, both the heat flux and the wall temperature are simultaneously controlled.

As shown in figure 6 and figure 7, in the case of combined ablative and radiative cooling, heat fluxes and wall temperature are successfully controlled for various permitted temperatures, when other cooling methods such as film cooling cannot reduce the wall temperature freely. This manifests the fact that cooling methods limit the propulsion system performances. It means that, according to the specifications of propellant, heat transfer limits thrust level (mass flow and pressure).

To select suitable TPS materials, comparison of mass and energy loss (Isp) is necessary. Figure 8 and figure 9 show the required TPS thicknesses and regressive rates for various permitted wall temperatures respectively.

These results also show that no ablation occurs if graphite was selected, but thicker insulator thicknesses would have been required, thereby leading to heavier TPS mass but lower energy loss and higher Isp. Figure 10 shows variation of TPS mass and Isp for 1000-s activity.

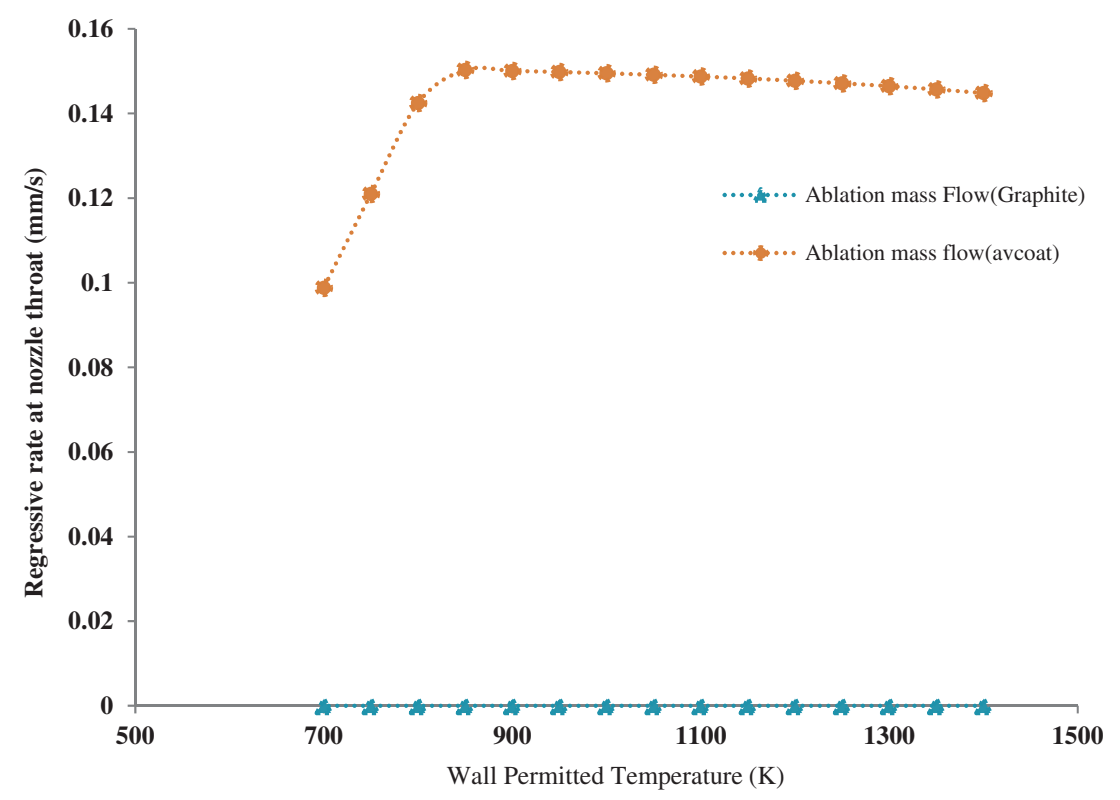

Figure 9. Comparison of regressive rates at nozzle throat for graphite and avcoat. 


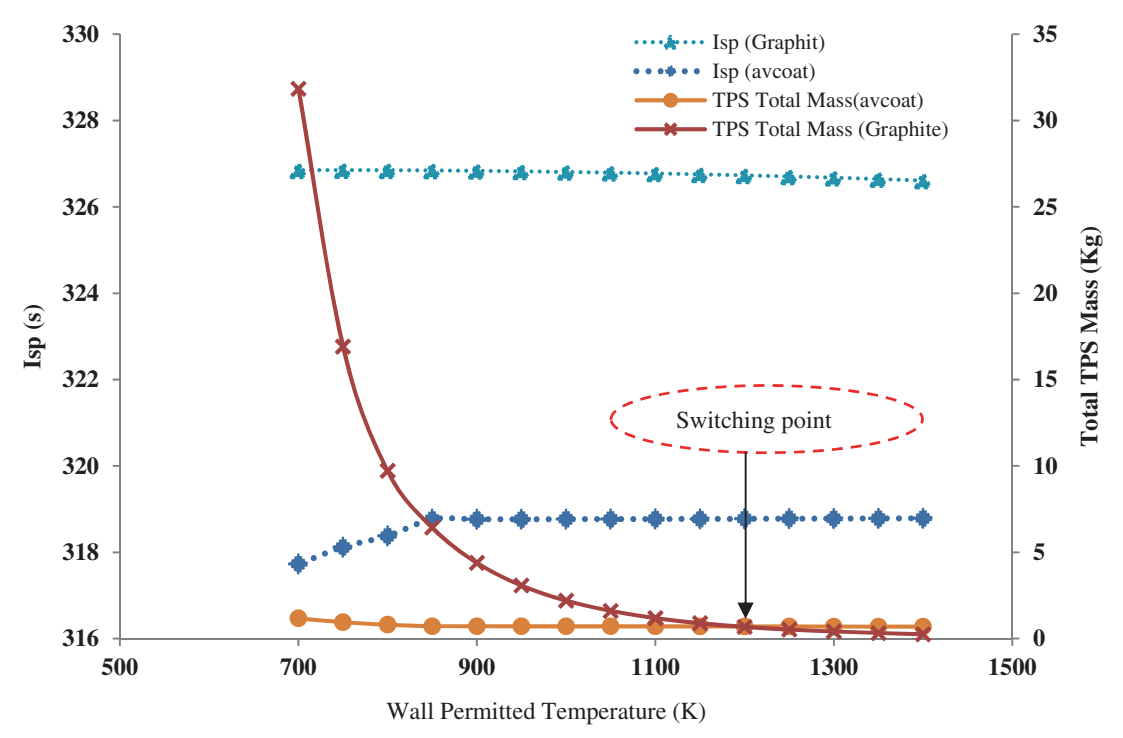

Figure 10. TPS mass and Isp variation for graphite and avcoat.

These results show that Isp variation is constant $(\sim 3 \%)$ but TPS mass variation depends on the wall temperatures (from 0 to 2700\%). In addition, TPS mass of avcoat is higher than graphite for wall temperatures higher than $1200 \mathrm{~K}$. Therefore, trading off between Isp and TPS mass should be done to derive the best selection of the insulator and ablative materials. In this case, it is proposed to select graphite for wall temperatures higher than $1200 \mathrm{~K}$ and avcoat for lower than $1200 \mathrm{~K}$. It should be noted that the results are true for $1000 \mathrm{~s}$ continuous firing time; for other firing times, similar analysis should be provided.

According to the results, it is possible to decrease the wall temperature to near $800 \mathrm{~K}$. Therefore, common materials can be applied with only 810 (gr) TPS mass (avcoat). The proposed algorithm can be used for every TPS and structure materials and decision making in selecting the best configuration by adding structure discipline to the proposed method.

\section{Summary and conclusion}

Controlling heat fluxes and wall temperature in steady state condition is the goal of every cooling method. Various cooling methods have been introduced for cooling of bipropellant thrusters, but it has been shown that utilization of convectional cooling methods limits the propulsion performance. In this paper, the combined ablative and radiative cooling is presented and the governing equations have been derived for two or three layers of TPS to optimize the TPS mass. The first layer is used as an ablative layer to control the temperature, where the second and third layers are used as an insulator to control the heat fluxes. To evaluate the proposed method, two real bipropellant thrusters were selected. It was shown that high temperature materials (platinum alloy) were needed for conventional cooling methods, which means higher cost. Using ablative and radiative cooling inside the thruster makes it possible to reduce the wall temperature to levels near the acceptable value of common materials such as steel alloys.

The proposed combined cooling method was applied to two real thrusters. According to the results, heat fluxes and wall temperature were successfully controlled for various permitted temperatures, when other cooling methods such as film cooling could not reduce the wall temperature freely. To select suitable TPS materials, mass and energy loss (Isp) tradeoffs were assessed. According to the results, selection of graphite (high ablation temperature) leads to higher Isp, but thicker insulator thicknesses are required. Selection of avcoat (low ablation temperature) leads to have lower Isp but lighter TPS mass. To choose the best solution, trading off between Isp and TPS mass was assessed.

Results of 1000-s burning time show that Isp variation is nearly constant but TPS mass variation depends on the wall temperature (from 0 to $2700 \%$ ). In addition, the required TPS mass of avcoat is higher than graphite for the wall temperature higher than $1200 \mathrm{~K}$. Therefore, it is proposed to select graphite for the wall permitted temperature higher than $1200 \mathrm{~K}$ and avcoat for the wall permitted temperature lower than $1200 \mathrm{~K}$.

Finally, the minimum required TPS mass is near 810-gr to reduce the wall temperature lower than $800 \mathrm{~K}$. The penalty of using TPS (mass penalty) is acceptable, considering the advantages of using steel alloy instead of platinum alloy.

\section{References}

[1] Dieter K Huzel and David H Huang 1992 Modern engineering for design of liquid propellant rocket engines. 370 L'Enfant Promenade, Washington DC, American Institute of Aeronautics and Astronautics 
[2] Mo Bai 2007 Numerical evaluation of heat transfer and pressure drop in open cell foams. MSc thesis, Florida, University of Florida

[3] Adami A 2015 Multidicsiplinary design optimization of Reentry Vehicle considering Guidance Algorithm. $\mathrm{PhD}$ thesis, Tehran, Iran, Amirkabir University of Technology

[4] Matteo Trotti 2011-2012 Modelling of liquid film cooling in a GOX/Kerosene rocket combustion chamber. $\mathrm{PhD}$ thesis, Politecnico di Milano

[5] Marco Pizzarelli, Francesco Nasuti and Marcello Onofri 2013 Trade-off analysis of high-aspect-ratio-cooling-channels for rocket engines, 44, pp. 458-467

[6] Torres Y, Stefanini L and Suslov D 2009 Influence of curvature in regenerative cooling system of rocket engine 1: 171184

[7] Mustafa Emre Boysan 2008 Analysis of regenarative cooling in liquid propellant rocket engines, MSc Thesis, Middle East Technical University

[8] Arnold R, Suslov D I and Haidn O J 2009 Film cooling of accelerated flow in a subscale combustion chamber. J. Propul. Power 25: 443-451

[9] Arnold R, Suslov D I and Haidn O J 2010 Film cooling in a high-pressure subscale combustion chamber. J. Propul. Power 26: $428-438$

[10] Hessam Babaee 2013 Analysis and optimization of film cooling effectivness, $\mathrm{PhD}$ Thesis, Louisiana State University

[11] Knab O, Frey M, Görgen J, Maeding C, Quering K and Wiedmann D 2009 Progress in combustion and heat transfer modelling in rocket thrust chamber applied engineering. Denver, Colorado, 45th AIAA/ASME/SAE/ASEE Joint Propulsion Conference \& Exhibit

[12] Hinckel J N, Savonov R I and Patire H 2011 Determination of thermal load in film cooled bipropellant thrust chambers by an inverse method, EUCASS 2011-421 experiments and test facilities, 2011. 4th European conference for aerospace sciences

[13] Heath Thomas Martin 2013 Assessment of the performance of ablative insulators under realistic solid rocket motor operating conditions, $\mathrm{PhD}$ thesis, The Pennsylvania State University

[14] David J Anderson, Michelle M Munk and John Dankanich 2009 Status and mission applicability of NASA's In-Space Propulsion Technology Project. Denver, Colorado, 45th AIAA/ASME/SAE/ASEE Joint Propulsion Conference \& Exhibit

[15] New Frontiers Program Library 2008 Advanced material bipropellant rocket (AMBR) information summary

[16] New Frontiers Program Library, [Online] Available at: http:// newfrontiers.larc.nasa.gov/NFPL.html

[17] Hossein Darabi, Jafar Roshanian and Hadi Zare 2014 Design of liquid-propellant engine using collaborative optimization and evolutionary algorithms, Proceedings of the Institution of Mechanical Engineers, Part G, Journal of Aerospace Engineering

[18] Ernst R L 2014 Development of a liquid bi-propellant rocket engine design, analysis and optimization tool. MSc thesis, Delft University of Technology, Faculty of Aerospace Engineering

[19] Amirhossein Adami, Mahdi Mortazavi and Mehran Nosratollahi 2015 A new approach in multidisciplinary design optimization of upper-stages using combined framework. ACTA Astronuatica 114: 174-183, September-October 2015 (doi: 10.5120/20496-2109)

[20] NASA Ames, Thermal protection materials and system branch TPSX Internet Database, (NASA TPSX website: http://asm.arc.nasa.gov)

[21] Nasa Space Vehicle Design Criteria August 1968 Entry thermal protection 